\title{
PREVENÇÃO DA TRANSMISSÃO VERTICAL DA SÍFILIS CONGÊNITA: PROMOÇÃO DA QUALIDADE DA ASSISTÊNCIA PRESTADA À PARTURIENTE
}

\author{
Benedita dos Santos Andrade ${ }^{1}$, Chennyfer Dobbins Abi Rached ${ }^{3}$ \\ 1 Graduanda de Enfermagem. Universidade Nove de Julho - UNINOVE. São Paulo - Brasil - Email: \\ São Paulo - Brasil \\ 2 Doutora em Saúde Coletiva; Mestre em Economia da Saúde pela Universidade Federal de São Paulo \\ (UNIFESP); Docente do Programa de Mestrado Profissional em Gestão em Sistemas de Saúde - \\ Universidade Nove de Julho - UNINOVE. São Paulo - Brasil email: chennyferr@yahoo.com.br
} ORCID: https://orcid.org/0000-0002-4499-3716

\section{RESUMO}

A sífilis consiste em uma doença de caráter infeccioso, crônica e sistêmica, causada pela bactéria Treponema pallidum, subespécie pallidum, podendo apresentar períodos de agudização e latência, quando não detectada e tratada de forma precoce. Identificar as ações de prevenção para a sífilis congênita no contexto da assistência pré-natal. Foi realizada uma pesquisa bibliográfica com consulta nas bases de dados LILACS, SCIELO, com seleção de material publicado entre 2013 e 2018. O período pré-natal é essencial para a implementação ações de promoção à saúde e prevenção de complicações tanto para a mãe como para o bebê, através do desenvolvimento de ações educativas, que devem ser desenvolvidas todas as etapas do ciclo gravídico-puerperal visando contribuir com uma gestação sem complicações, e com o apoio físico e psicológico da gestante. Foi constatado com este estudo que a sífilis ainda representa um grave problema de saúde e que se não detectada precocemente, pode ser transmitida para o bebê. E é nesse contexto que se ressalta a importância do pré-natal, para acompanhar as gestantes que visa estabelecer medidas de cuidados e controle de diversas doenças ou complicações, incluindo a sífilis, pois através desse acompanhamento já nas primeiras consultas, é preconizado o exame de rastreamento da doença, havendo, portanto, melhores chances de reduzir os riscos para o bebê, com uma identificação e tratamento precoce.

Palavras-chaves: Gestação, Pré-Natal, Sífilis Congênita, Assistência de Enfermagem.

\begin{abstract}
Syphilis consists of a chronic and systemic infectious disease caused by the bacterium Treponema pallidum, subspecies pallidum, and may present periods of exacerbation and latency when not detected and treated early. To identify prevention actions for congenital syphilis in the context of prenatal care. A bibliographic research was conducted with consultation in the databases LILACS, SCIELO, with selection of material published between 2013 and 2018. The prenatal period is essential for the implementation of health promotion actions and prevention of complications for both mother and the baby, through the development of educational actions, that must be developed all stages of the pregnancy-puerperal cycle aiming to contribute to a pregnancy without complications, and with the physical and psychological support of the pregnant woman. It was found in this study that syphilis still represents a serious health problem and that if not detected early, can be transmitted to the baby. It is in this context that the
\end{abstract}


importance of prenatal care is emphasized to accompany the pregnant women, who aim to establish measures of care and control of various diseases or complications, including syphilis, because through this follow-up already in the first consultations, therefore better chances of reducing risks to the baby, with early identification and treatment.

Key words: Pregnancy, Prenatal Care, Congenital Syphilis, Nursing Care.

\section{INTRODUÇÃO}

As Doenças Sexualmente Transmissíveis representam um grave problema de saúde pública, em âmbito mundial associada ainda a várias limitações sociais, econômicas, e sanitárias. Estimativas apontam que possam surgir aproximadamente 340 milhões de novos casos de DST no mundo, e que desse número, cerca de 12 milhões, se referem à sífilis na população a, e que 98\% dos casos ocorram em países em desenvolvimento. Somente na América Latina mais de 3 milhões de pessoas são afetadas. Sendo assim, embora seja uma doença passível de prevenção, e de modo geral, tratamentos acessíveis, os índices ainda são elevados em todo o mundo ${ }^{1,2,3}$.

A sífilis consiste em uma doença de caráter infeccioso, crônica e sistêmica, causada pela bactéria Treponema pallidum, subespécie pallidum, podendo apresentar períodos de agudização e latência, quando não detectada e tratada de forma precoce. Quanto a sua transmissão esta pode ser feita por via sexual ou vertical, sendo esta última quando a mãe passa para o feto, passando a ser classificada como sífilis adquirida e sífilis congênita ${ }^{1,4}$.

Dessa forma, a sífilis congênita ocorre devido a disseminação hematogênica da bactéria da doença que está na gestante infectada não tratada, para feto por via transplacentária, por esse motivo, dá-se o nome de transmissão vertical, contudo, ainda há a possibilidade de transmissão direta pelo contato do recém-nascido com lesões no canal do parto ${ }^{5}$.

Estimativas apontam, que mais dois milhões de mulheres grávidas são afetadas durante a gravidez, o que contribui com taxas de até $25 \%$ de natimortos ou abortos espontâneos e $25 \%$ nascidos de baixo peso ou infecções graves. Os fatores de riscos relacionados incluem: baixo nível socioeconômico, automedicação, baixa escolaridade, promiscuidade e precocidade sexual, e, além disso, a falta de um acompanhamento adequado no período pré- natal ${ }_{1,5}$.

Frente a alta incidência e risco tanto para a mulher como para o bebê, a assistência pré-natal é fundamental para o acompanhamento de ambos, além de ser embasada pelo Programa de Humanização no Pré-Natal e Nascimento (PHPN), que dentre outros objetivos visa garantir 
melhora do aceso e qualidade do pré natal, assistência ao parto e puerpério tanto para as mulheres como para os recém-nascidos ${ }^{6}$.

Sendo assim, a Assistência pré-natal deve ser considerada como uma ferramenta para a prevenção, detecção precoce, tratamento e redução dos índices de morbidades relacionadas a sífilis congênita, desde que, seja realizada de forma correta, alcance as populações mais extremas da sociedade, principalmente as mais vulneráveis, contribuindo ainda com a qualidade da assistência prestada ${ }^{6}$.

Frente ao exposto surge a seguinte indagação: Como deve ser feita a prevenção vertical da sífilis congênita de forma que promova a qualidade da assistência prestada à parturiente? A escolha do tema se justifica, frente à alta incidência da sífilis congênita, e principalmente pelos riscos que esta doença representa tanto para a mulher como para o bebê, contudo, representa uma doença passível de tratamento desde que diagnosticada de forma precoce, e assim, enfatiza-se a importância do pré-natal para que esse objetivo seja alcançado. Espera-se, portanto, que a discussão dessa temática venha contribuir com ampliação dos conhecimentos em relação a essa importância do acompanhamento pré-natal na redução dos índices de sífilis congênita, garantindo assim, uma assistência integral e de qualidade tanto à gestante quanto ao bebê.

\section{OBJETIVO}

Identificar as ações de prevenção para a sífilis congênita no contexto da assistência pré-natal

\section{METODOLOGIA}

Foi realizada uma pesquisa bibliográfica desenvolvida com base em material já elaborado, constituído principalmente de livros e artigos científicos, e que consiste em um procedimento reflexivo, sistemático, controlado e crítico, que favorece o descobrimento de novos fatos ou dados, relações ou leis, em qualquer campo de conhecimento, que se caracteriza, como o primeiro passo do pesquisador para realizar uma pesquisa científica, sendo possível por meio desse processo, fazer levantamentos dos dados já existentes em material publicado, constituído principalmente por livros-texto, artigos científicos, dissertações, teses, entre outros, devendo, portanto, seguir as seguintes fases: escolha do tema, elaboração do trabalho, identificação, localização, compilação, fichamento, análise e interpretação e redação ${ }^{7,8}$.

Para a realização da pesquisa foram consultadas as seguintes bases de dados informatizadas: Literatura Latino Americana e do Caribe em ciências da saúde (LILACS), Scientific Eletronic Library On line, (SCIELO), disponíveis na Biblioteca Virtual em Saúde (BVS), e Bibliotecas Integradas da Universidade de São Paulo. 
Os critérios de inclusão dos artigos foram estabelecidos da seguinte maneira: ser artigo de pesquisa publicado em periódicos nacionais, na língua portuguesa, e hispânica, indexados em bases de dados e publicados no periódico de 2013 a 2018. Como critérios de exclusão, serão consideradas publicações com data anterior a 2013, que não da língua portuguesa e hispânica, e que indexada somente o resumo.

Para tanto foram utilizadas as seguintes Palavras-chave: Gestação, Pré-Natal, Sífilis Congênita, Assistência de Enfermagem.

Para síntese e análise do material foram realizados os seguintes procedimentos: Leitura informativa ou exploratória, que constitui na leitura do material para saber do que se tratavam os artigos; leitura seletiva, que se preocupou com a descrição e seleção do material quanto a sua relevância para o estudo; leitura crítica e reflexiva que buscou por meio dos dados a construção dos resultados encontrados.

\section{DESENVOLVIMENTO}

\subsection{Pré-natal: acesso de cuidados para a gestante}

O período pré-natal é essencial para a implementação ações de promoção à saúde e prevenção de complicações tanto para a mãe como para o bebê, através do desenvolvimento de ações educativas, que devem ser desenvolvidas todas as etapas do ciclo gravídico-puerperal visando contribuir com uma gestação sem complicações, e com o apoio físico e psicológico da gestante $^{9,10}$.

A Atenção Básica representa o primeiro nível de atenção, é, portanto, a porta de entrada do usuário ao sistema de saúde, tendo como objetivos, organizar e integrar as redes de atenção à saúde e à promoção, prevenção e recuperação da saúde. Dentre as possibilidades de acesso a essa assistência, destaca-se, a assistência pré-natal, que deve favorecer, dentre outros aspectos: a realização da primeira consulta pré-natal o mais precoce possível; a garantia de, no mínimo, seis consultas pré-natais, sendo indicado, uma no primeiro trimestre, duas no segundo trimestre e três no terceiro trimestre da gestação; a realização de todos os exames laboratoriais preconizados, inclusive, exames para diagnóstico da sífilis, com uma coleta na primeira consulta e outra no terceiro trimestre de gestação ${ }^{10}$.

A assistência pré-natal, é uma oportunidade para que se desenvolva ações para o controle e prevenção da Sífilis Congênita, onde os profissionais podem realizar a triagem de sífilis, como uma rotina para todas as mulheres, e seus parceiros. Além disso, ao ser identificado esses casos, é preciso que haja a notificação na vigilância epidemiológica visando manter os registros de 
ocorrências e controle sempre atualizados, que por sua vez, também irão contribuir com o desenvolvimento de estratégias ${ }^{9,10}$.

\subsection{Ações de prevenção para a sífilis congênita no contexto da assistência pré-natal}

Para o controle e prevenção da sífilis congênita, no contexto pré-natal, são sugeridas medidas simples, que podem representar ainda o cuidado humanizado, tanto as mães como aos recémnascidos. O período pré-natal, é considerada um momento de grande importância para a transmissão dessas informações, uma vez que, os níveis de consultas pré-natais conforme preconizado são altos; os testes de detecção são considerados de baixo custo, além do fato d que, podem ser realizados no nível de cuidados primários de saúde; o tratamento com penicilina também é de baixo custo; e o medicamento está na lista dos medicamentos essenciais de todos os países, mas, embora esse fator sejam favoráveis para a prevenção da sífilis, esta doença ainda representa um grave problema de saúde pública ${ }^{11}$.

Contudo, é possível mudar essa realidade, a partir das ações dos profissionais envolvidos nesse tipo de assistência, quando este buscarem desenvolver de forma efetiva, ações educativas, que abordem a temática, incentivando, principalmente medidas de prevenção, além de capitação das gestantes para iniciarem de forma precoce o pré-natal; realização de uma assistência prénatal de qualidade e organizada; instituição do tratamento adequado da gestante e do(s) seu(s) parceiro(s), além da abordagem clínica, e epidemiológica, dos casos identificados, buscando ainda identificar os casos de sífilis congênita. ${ }^{11,12}$.

Frente a alta incidência da sífilis, no Brasil, esta foi incluída na lista de doenças e agravos de notificação compulsória pela Portaria GM/MS $n^{0}$ 33/2005. Para fins de vigilância epidemiológica usa-se a definição de ${ }^{12}$ :

Gestante que apresente teste não treponêmico reagente com qualquer titulação e teste treponêmico reagente, independente de qualquer evidência clínica de sífilis, realizados durante o pré-natal; e gestante com teste treponêmico reagente e teste não treponêmico não reagente ou não realizado, sem registro de tratamento prévio. (Ramalho 2016, p.21).

Sendo assim, é imprescindível, que o teste para sífilis seja realizado em todas as mulheres, durante a assistência pré-natal, já na primeira consulta e no terceiro trimestre gestacional, que pode ser realizado por meio do TR de sífilis e do Veneral Disease Research Laboratory (VDRL). O TR de sífilis refere-se a um tipo de teste treponêmico, qualitativo, usado como triagem para determinar a reatividade de uma amostra de sangue pela busca de anticorpos específicos para o Treponema pallidum. Já oVDRL é um teste não treponêmico, quantitativo, 
que detecta anticorpos não específicos para o T. pallidum sendo este, mais utilizado para determinar os títulos dos anticorpos presentes nas amostras reagentes nos testes treponêmicos, como o TR, além de poder contribuir com o monitoramento da resposta ao tratamento ${ }^{12}$.

Quanto a recomendação da realização do exame já na primeira consulta de pré-natal, é respalda pelo MS, e se este for negativo, é preciso que se repita a testagem com o VDRL no terceiro trimestre gestacional. Se ocorrer a positividade já no primeiro mês $\mathrm{cm}$ TR, a recomendação é que seja realizado imediatamente a coleta do VDRL, e a testagem dos parceiros sexuais, que por sua vez, dando positivo, confirma-se a infecção, e já é preciso estabelecer o tratamento para a mulher e seu parceiro. Após a realização do tratamento com penicilina, conforme indicações, é preciso que a mulher seja acompanhada mensalmente com o VDRL ${ }^{10,12}$.

Na prevenção da sífilis congênita, é, portanto, fundamental que haja o rastreamento precoce da infecção, que pode ser obtida através da realização também precoce do teste rápido e/ou VDRL, tratamento adequado da mulher e parceiro, além do controle de cura de todos os casos de sífilis identificados durante o pré-natal ${ }^{10,12}$.

Sendo assim, uma das principais ações preventivas, destacada em vários estudos se refere-se à é a triagem sorológica, da mãe na maternidade, uma vez que, a mãe ao adquirir sífilis durante a gravidez, pode desencadear a infecção assintomática ou sintomática nos recém-nascidos, estudos apontam que até $50 \%$ das crianças recém-nascidas tenham a infecção de forma assintomática, e os primeiros sintomas ocorrem geralmente nos primeiros três meses de vida. 11,12 .

Para detecção precoce da sífilis congênita, é recomendado o exame, físico, a coleta VDRL no segundo ou terceiro dia de vida, além da sorologia no líquor (Líquido Cefalorraquidiano) que deve ser realizada para verificação de neurosífilis, em todo recém-nascido de mãe acometida pela doença. Havendo o aumento das células acima de 25 ou das proteínas acima de 150 no resultado do exame estes podem indicar a neurosífilis. Em alguns caos são realizados radiografias de ossos longos para verificar as alterações radiológicas que podem ocorre, principalmente quando os recém-nascidos são sintomáticos. Os locais mais afetados são as metáfises e diáfises dos ossos longos, onde podem surgir lesões são simétricas e tendem a serem múltiplas ${ }^{10,11}$.

O tratamento do recém-nascido em caso de alterações que sugiram a sífilis congênita, deve seguir alguns parâmetros, conforme mostrado no quadro ${ }^{9-13}$. 
Quadro 1: Alterações bebê- Indicação da Sífilis.

\begin{tabular}{|c|c|}
\hline Na presença de & Tratamento \\
\hline $\begin{array}{c}\text { alterações clínicas e/ou sorológicas } \\
\text { e/ou radiológicas }\end{array}$ & $\begin{array}{l}\text { penicilina cristalina } 50.000 \mathrm{UI} / \mathrm{kg} / \mathrm{dose}, \mathrm{EV} \text {, duas vezes ao dia se tiver } \\
\text { menos de uma semana de vida e três vezes ao dia se tiver mais de uma } \\
\text { semana de vida, por } 10 \text { dias; } \\
\text { ou penicilina } \mathrm{G} \text { procaína } 50.000 \mathrm{UI} / \mathrm{kg} \text {, IM, por } 10 \text { dias. }\end{array}$ \\
\hline alteração liquórica, & $\begin{array}{c}\text { Penicilina G cristalina, } 50.000 \mathrm{UI} / \mathrm{kg} / \text { dose, } \mathrm{EV} \text {, duas vezes por dia se } \\
\text { tiver menos de uma semana de vida e três vezes ao dia se tiver mais de } \\
\text { uma semana de vida, por } 14 \text { dias }\end{array}$ \\
\hline $\begin{array}{c}\text { alterações clínicas, radiológicas } \\
\text { e/ou liquóricas, e a sorologia do RN } \\
\text { for negativa, }\end{array}$ & $\begin{array}{l}\text { penicilina G benzatina, IM, na dose única de 50.000UI/kg } \\
\text { O acompanhamento é obrigatório; sendo impossível o } \\
\text { acompanhamento, o RN deve ser tratado com o esquema }\end{array}$ \\
\hline $\begin{array}{l}\text { Se o RN não for reagente ou for } \\
\text { reagente com titulação menor ou } \\
\text { igual à materna e também for } \\
\text { assintomático e com radiografia de } \\
\text { ossos longos sem alterações }\end{array}$ & fazer apenas seguimento ambulatorial. \\
\hline
\end{tabular}

O não acompanhamento adequado da mulher durante o pré-natal, que vise o controle da SC, pode resultar em complicações, tanto para a mulher como para ao bebê, incluindo maior probabilidade de aborto e malformação congênita. O simples fato de não notificação da sífilis, quando identificada, se configura como um agravante, para a controle dessa ocorrência, frente a essa realidade, é de fundamental importância, que se busque a identificação precoce de casos de sífilis materna e congênita em serviços de pré-natal e em maternidades, para que haja o desenvolvimento de ações de prevenção e tratamento ${ }^{14,15}$.

Sob esta perspectiva, um estudo reforça que, a identificação da sífilis, assim como seu manejo clínico e terapêutico, se configura como simples e fácil de realizar, sendo, portanto, uma doença de fácil controle, desde que haja uma assistência pré-natal de qualidade. Contudo, na prática, não tem ocorrido a devida valorização para essa assistência como uma forma de prevenir a SC, o que tem contribuído, com a baixa de medidas de prevenção da doença, bem como, aumento dos $\operatorname{casos}^{9,11}$.

Visando ampliar as estratégias para controle da SC, além do PHPN, em 2007 foi lançado o Plano Nacional de Redução da Transmissão Vertical do HIV e da Sífilis, cuja a meta na época era de reduzir as taxas de transmissão mãe-filho do HIV e da sífilis até 2011. Contudo, essa meta não foi alcançada ao se analisar altos índices dessa ocorrência ainda nos dias de hoje. Desde a implementação desse plano o MS, vem buscando desenvolver ações que priorizem a atenção à gestante e bebê, durante todo período neonatal, de forma humanizada, destacandose o Projeto Rede Cegonha, que representa uma estratégia que busca garantir o direito à mulher, ao planejamento reprodutivo e à atenção humanizada à gravidez, ao parto e ao puerpério, bem 
como à criança o direito ao nascimento seguro e ao crescimento e ao desenvolvimento saudáveis, refletindo diretamente na redução dos índices de mortalidade materna no país ${ }^{13,15}$.

Se faz importante observar que, para uma assistência que resulte na redução de casos de sífilis congênita, é preciso considerar o parceiro da gestante, o que nem sempre é comtemplado pelos profissionais, devido à ausência deste, o que torna um desafio para os sistemas de saúde. Uma das estratégias utilizadas por enfermeiras, em um estudo, foi enviar comunicado pela própria mulher, para que o parceiro compareça nos serviços de saúde, e quando isso não corria, eram enviados até a sua residência, agentes comunitários para a realização da visita domiciliar. Contudo, relata-se ainda que mesmos e utuilizando dessas estratégias, nem sempre é possível o contato, com o parceiro, o que acaba fragilizando as ações de prevenção ${ }^{15,16}$.

\section{CONSIDERAÇÕES FINAIS}

Foi constatado com este estudo que a sífilis ainda representa um grave problema de saúde e que se não detectada precocemente, pode ser transmitida para o bebê. E é nesse contexto que se ressalta a importância do pré-natal, para acompanhar as gestantes que visa estabelecer medidas de cuidados e controle de diversas doenças ou complicações, incluindo a sífilis, pois através desse acompanhamento já nas primeiras consultas, é preconizado o exame de rastreamento da doença, havendo, portanto, melhores chances de reduzir os riscos para o bebê, com uma identificação e tratamento precoce.

Contudo, se faz necessário ampliar a consciência das mulheres quanto a importância da frequência das consultas pré-natal conforme preconizada, e nesse cenário, os profissionais de enfermagem que atuam diretamente com a mulher, durante todo período gravídico-puerperal, têm condições de abordar essas mulheres promovendo sua conscientização através de ações educativas.

\section{REFERÊNCIAS}

1. Calás JES. Sífilis gestacional em municípios selecionados da Região Metropolitana I do Estado do Rio de Janeiro, 2011 a 2013. 66f. 2015 [dissertação]- Escola Nacional de Saúde Pública Sérgio Arouca - Instituto Fio Cruz[internet]. Rio de Janeiro, 2015. Disponível em: bvssp.icict.fiocruz.br/lildbi/docsonline/get.php?id=4316.

2. Saraceni V, Miranda, AE. Relação entre a cobertura da Estratégia Saúde da Família e o diagnóstico de sífilis na gestação e sífilis congênita. Cad. Saúde Pública[internet]. 2012; 28(3):490-6. Disponível em: http://www.scielo.br/scielo.php?pid=S0102311X2012000300009\&script=sci_abstract\&tlng=pt. 
3. Ministério da Saúde. Secretaria de Vigilância em Saúde (BR). Programa Nacional de DST e Aids. Boletim Epidemiológico Aids e DST. Brasília: Ministério da Saúde [internet]. 2012. Disponível em: http://www.aids.gov.br/pt-br/centrais-de-conteudos/boletins-epidemiologicosvertical.

4. Magalhães DMS, Kawaguchi IAL, Dias A, Calderon IMP. Sífilis materna e congênita: ainda um desafio. Cad. Saúde Pública [internet]. 2013; 29(6), 1109-20. Disponível em: http://www.scielo.br/scielo.php?script=sci_arttext\&pid=S0102-311X2013000600008.

5. Clemente TS, Lima MM. A importância do pré-natal como ferramenta na prevenção da sífilis congênita: revisão bibliográfica. Cadernos de Graduação - Ciências Biológicas e da Saúde Fits[internet]. 2012; 1(1):33-42. Disponível em: https://periodicos.set.edu.br/index.php/fitsbiosaude/article/view/455.

6. Suto CSS, Silva DL, Almeida ES, costa LE, Evangelista TJ. Assistência pré-natal a gestante com diagnóstico de sífilis pré-natal. Rev Enferm Atenção Saúde [internet]. 2016; 5(2):18-33. Disponível em: http://seer.uftm.edu.br/revistaeletronica/index.php/enfer/article/view/1544. Acesso em 23 mai 2018.

7. Medeiros, JB. Redação Científica: a prática de fichamentos, resumos, resenhas. 12. Ed. São Paulo: Atlas, 2017.

8. Severino A J. Metodologia do Trabalho Científico. 24 ed. São Paulo: Cortez, 2016.

9. Calás JES. Sífilis gestacional em municípios selecionados da Região Metropolitana I do Estado do Rio de Janeiro, 2011 a 2013. FioCRUZ, 2016. Disponível em: https://www.arca.fiocruz.br/handle/icict/13477.

10. Rezende EMA, Barbosa NB. A sífilis congênita como indicador da assistência de pré-natal no estado de goiás. Rev. APS. [internet]. 2015 [acesso 01 out 2018]; 18(2): 220 - 232. Disponível em: https://aps.ufjf.emnuvens.com.br/aps/article/view/2421.

11. Sousa DMN, Costa CC da, Chagas ACMA. Sífilis congênita: reflexões sobre um agravo sem controle na saúde mãe e filho. Rev Enferm UFPE Recife[internet].2014 [acesso 01 out 2018]; 8(1):160-5. Disponível em:

https://periodicos.ufpe.br/revistas/revistaenfermagem/article/download/9619/96.

12. Ramalho MOL. Avaliação da assistência pré-natal com ênfase na sífilis gestacional na estratégia de saúde da família do Recife. 96f [dissertação] - Universidade Federal de Pernambuco, 2016. Disponível em: https://repositorio.ufpe.br/handle/123456789/17410. 
13. Costa CC, Freitas LV, Sousa MN, Oliveira LL, Chagas ACM, Lopes MVO. Sífilis congênita no Ceará: análise epidemiológica de uma década. Rev Esc Enferm USP 2013; 47(1):152-9. Disponível em: http://www.scielo.br/scielo.php?script=sci_arttext\&pid=S008062342013000100019 .

14. Secretária de Saúde. Prevenção da transmissão vertical do HIV, sífilis congênita e hepatites B e C: Guia para maternidades. Secretaria Estadual de Saúde do Rio Grande do Sul, 2016. Disponível em: http:/www.saude.rs.gov.br/upload/arquivos/201703/16151232-4-5-2-guiamaternidades.pdf.

15. Figueiredo MS, Cavalcante EGR, Oliveira CJ, Monteiro MFV, Quirino GS, Oliveira DR. Percepção de enfermeiros sobre a adesão ao tratamento dos parceiros de gestantes com sífilis. Rev Rene. [internet]2015 [acesso 10 out 2018 16(3):345-54. Disponível em: http://www.periodicos.ufc.br/rene/article/view/2789.

16. Nunes JT, Marinho AC, Davim RMB, Silva GGO, Feliz RS, Martino MMF. Sífilis na gestação: perspectivas e condutas do enfermeiro. Rev enferm UFPE [internet].2017 [acesso 01 out 2018]; Recife, 11(12):4875-84. Disponível em: https://periodicos.ufpe.br/revistas/revistaenfermagem/article/viewFile/23573/25297 